\title{
Robô para Reconstrução Tridimensional: Uma aplicação didático-pedagógica do protótipo no âmbito da Engenharia e Computação
}

\author{
João L. de S. Silva ${ }^{1}$, Michelle M. Cavalcante ${ }^{1}$, Fabiano A. Vaz ${ }^{2}$, Esdriane C. Viana ${ }^{1}$ \\ ${ }^{1}$ Departamento de Engenharia Elétrica - Instituto Federal da Bahia (IFBA) \\ Caixa Postal 15.064 - 91.501-970 - Paulo Afonso - BA - Brasil
}

${ }^{2}$ Centro de Informática - Universidade Federal de Pernambuco (UFPE) - Recife, PE Brasil

\{jlucas.silva,michellemelo.c, esdriane\}@ifba.edu.br, fav2@cin.ufpe.br

\begin{abstract}
This study aims to show the educational application of a robot prototype to assist in three-dimensional reconstruction. For this, we used the Arduino platform and through this was set up a controlled robot for a notebook with a Kinect. The tool presented potentialities that are considered relevant in the teaching-learning process, especially in multidisciplinary areas, ensuring an incentive to learning according to the students, as well as providing the three-dimensional reconstruction, that also be used in the teaching process, as example, the use of $3 D$ reconstructions of electrical insulators.
\end{abstract}

Keywords. Robotics, Education, RecArd, Arduino.

Resumo. O presente trabalho tem por finalidade mostrar a aplicação educacional de um protótipo de Robô para auxiliar na reconstrução tridimensional. Para tanto, utilizou-se a plataforma Arduino e através desta montou-se um robô controlado por um notebook junto a um Kinect. A ferramenta apresentou potencialidades que são consideradas relevantes no processo de ensino-aprendizagem, principalmente, em áreas multidisciplinares, o que garantiu um incentivo ao aprendizado de acordo com os estudantes, além de propiciar a reconstrução tridimensional que também pode ser utilizada no processo de ensino, a exemplo do uso de reconstruções $3 D$ de isoladores elétricos.

Palavras-chaves. Robótica, Educação, RecArd, Arduino.

\section{Introdução}

A Robótica Educacional é uma importante ferramenta multidisciplinar, motivacional e lúdica, o que torna um exímio método para auxiliar no processo de ensino-aprendizagem, pois possibilita a inserção do estudante na cultura digital e transforma a informação em conhecimento das mais variadas áreas da ciência.

No mundo atual, a necessidade de renovação é um fenômeno social que ocorre em escala global. Dentro da área pedagógica, seguindo o paradigma de mudança defendido por Cassol (2000), a educação também está acompanhando essa evolução tecnológica, o 
que proporcionou o surgimento do conceito de "novas tecnologias educacionais" [Levy $\&$ da Costa 1993]. Essas tecnologias educacionais mais atuais e o uso de robôs em sala de aula, recebe uma nova denominação: robótica educacional. Essa pratica constitui-se numa ferramenta investigativa e lúdica onde se emprega a criatividade do discente na criação de soluções de hardware e software visando a resolução de um desafio. [Barros et al. 2014].

Alinhado com a teoria construcionista defendida por Piaget, Papert (2008) propõe a ideia de que os seres humanos aprendem melhor quando são envolvidos no planejamento e na construção dos objetos. Nessa circunstância, a Robótica Educacional ganha força por se tratar da aplicação da robótica na área pedagógica, com o objetivo de disponibilizar aos alunos a oportunidade de criar soluções voltadas ao mundo real, possibilitando o aprendizado de forma dinâmica e estimulante. Tal aplicação é capaz de unir atividades de mecânica, como, por exemplo, a construção dos objetos controlados e atividades de raciocínio lógico, que envolvem cálculos de circuitos eletrônicos e desenvolvimento de programas que visam fazer com que determinado circuito eletrônico cumpra as atividades especificadas. [Santos et al. 2010]

Desta forma, percebe-se o grande potencial da robótica educacional como ferramenta multidisciplinar, visto que, a construção de protótipos em geral faz com que o aluno questione e seja capaz de relacionar diferentes conhecimentos e aptidões de forma a solucionar um problema. A busca por soluções estimula o espírito investigativo, fortemente motivado pela curiosidade, e permite que o aluno extrapole os conhecimentos individuais de cada disciplina. Assim a robótica assume o papel de uma ponte de ligação interdisciplinar visando a construção do conhecimento coletivo através da aplicação com a realidade. [Santos et al. 2010. Adaptado]

Frente a este cenário, o presente trabalho montou um Robô para reconstrução tridimensional a partir da plataforma de prototipagem Arduino e aplicou o protótipo como um método para auxiliar e complementar o ensino-aprendizagem. $\mathrm{O}$ trabalho mostrou aos discentes de forma mais ampla assuntos voltados para computação e engenharia para que estes associassem a disciplina com o seu cotidiano. Para mais, o projeto pretende estimular o estudante a pensar, a aguçar a sua curiosidade científica e a perceber os benefícios da robótica educacional para o desenvolvimento e aprimoramento da sua capacidade de memorização, raciocínio, concentração e atenção.

O restante do trabalho está organizado como segue. A seção 2 apresenta conceitos e informações do projeto e ferramentas, a seção 3 aborda os materiais e métodos, a seção 4 expõe uma discussão acerca do assunto, apresenta e analisa resultados, por fim, a seção 5 apresenta as conclusões obtidas com o projeto.

\section{Arduino, RecArd e Reconstrução 3D}

Este capítulo traz uma visão geral do projeto Arduino, sua história e informações sobre licença e funcionamento, além do RecArd, um robô criado com o intuito de demostrar a facilidade de se trabalhar com esse tipo de projeto e como forma de incentivar alunos do ensino técnico e superior, e o funcionamento da Reconstrução Tridimensional. 


\subsection{Arduino}

A plataforma Arduino surgiu com o objetivo de criar um dispositivo para controlar projetos construídos de uma forma mais acessível e simples do que outros sistemas disponíveis no mercado [Silva et al. 2014a]. A plataforma Arduino, é open-source baseada em hardware e software para as áreas de automação e robótica. Ou seja, pode-se utilizar comercialmente e pessoalmente, além de permitir obras derivadas [Cavalcante et al. 2014].

O Arduino utiliza um Microcontrolador ATMEGA (chip controlador gravável) com a função de receber e entregar o fluxo de informações com o controle por software [Silva et al. 2014a].

\subsection{RecArd}

O RecArd é um Robô com um ambiente computacional cujo objetivo é realizar reconstruções tridimensionais de forma fácil, lúdica e interativa. O sistema desenvolvido é um protótipo capaz de encontrar objetos que se deseja reconstruir tridimensionalmente de forma automática, sendo ideal para exploração de lugares de difícil acesso.

No protótipo atual, a exploração de ambientes pelo Robô como cavernas com solo desnivelado e com obstáculos ainda não é completamente aplicável e eficaz devido à fraca potência dos motores e a ausência de suspensão nas rodas, pois são fatores de aumento no custo do protótipo. Entretanto, tais fatores não são limitantes para a continuação do projeto.

Ciente de que o foco principal deve ser o conteúdo e as possibilidades que o projeto demonstra, o Robô foi elaborado e testado em residências e laboratórios, e assim, coletado resultados do algoritmo no protótipo de baixo custo. Vale destacar a aplicação educacional que se conseguiu antes de inserir o Kinect ${ }^{1}$ [Silva et al. 2014b].

A aplicação educacional do Robô foi algo destaque, pois pode-se ensinar eletrônica, linguagem de programação, mecânica, além de outros conceitos com o protótipo, fora a possibilidade de reconstruir objetos tridimensionalmente para mostrar em sala de aulas, como isoladores e máquinas elétricas.

\subsection{Reconstrução 3D}

A reconstrução de modelos tridimensionais (3D) consiste na criação de uma superfície ou malha poligonal, obtida através da interpolação de pontos amostrados, no caso do kinect, é obtida as informações de profundidade contidas nas imagens e elaborado uma matriz de pontos (nuvem de pontos), conforme a Figura 1, processo feito pelo algoritmo ICP (Iterative Closest Point) e o uso do Kinect Fusion [Silva et al. 2014a].

\footnotetext{
${ }^{1}$ Kinect: Dispositivo da Microsoft confeccionado com o objetivo de melhorar a interação homem-máquina destinado, inicialmente, ao console de jogos Xbox 360.
} 


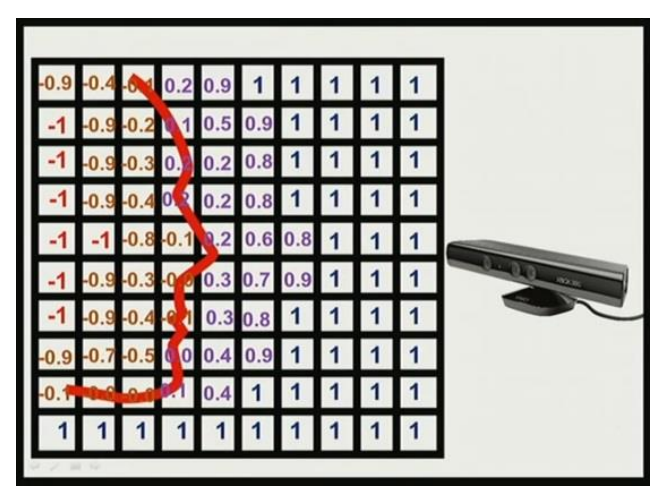

Figura 1. Exemplo de uma Matriz formada com o Emissor IR e Sensor IR.

O hardware do kinect é constituído por uma câmera RGB (Sensor RGB), emissor infravermelho (Emissor IR), câmera de infravermelho (Sensor IR) e microfones. A câmera e o sensor funcionam na frequência de $30 \mathrm{~Hz}$, com resolução de 640 x 480 pontos, conforme a Figura 2. São localizados os pontos cuja distância disposta está entre 70 centímetros até 6 metros.

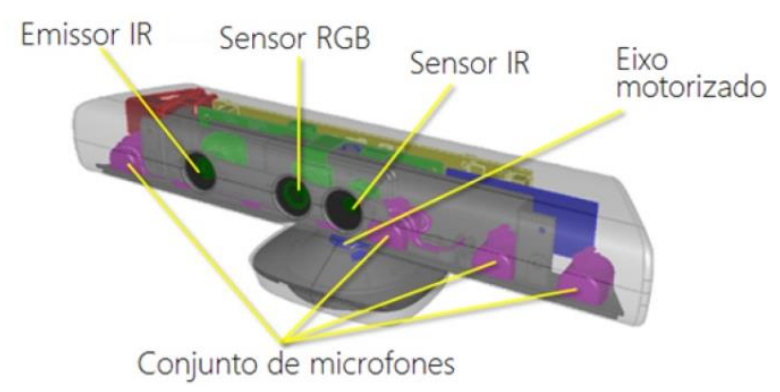

Figura 2. Hardware do Kinect [Cardoso 2013].

Ao terminar o tempo (delay) destinado e configurado para reconstrução 3D, o objeto é salvo e o Robô pode voltar para o ponto inicial, antes do processo de localização do objeto ou tentar localizar outro item.

\section{Materiais e métodos}

O Robô foi desenvolvido para funcionar integrado entre um conjunto de tecnologias. O ambiente é composto por um pequeno chassi controlado de dois modos: à distância pelo usuário via acesso remoto ou no modo automático com a atuação de sensores ultrassônicos. Com a ajuda do sensor de profundidade e o sensor RGB do Kinect, o Robô consegue localizar um objeto quando adicionado um modelo em sua biblioteca e depois que encontrá-lo, realiza a reconstrução 3D do mesmo com um algoritmo ICP (Iterative Closest Point).

Os materiais utilizados para construção do robô estão relacionados na Tabela 1. Além do Arduino, destaca-se o uso do Motor Shield L293d que serviu para possibilitar o uso dos motores DC (Motor de Corrente Contínua) para realizar o giro das rodas para frente e para trás. 
Tabela 1 - Materiais para a elaboração do RecArd.

\begin{tabular}{c|c}
\hline QUANTIDADE & MATERIAIS \\
\hline 01 & Placa Arduino UNO R3 \\
\hline 01 & Mini-Protoboard \\
\hline 04 & Motores DC com caixa de Redução \\
\hline 04 & Rodas de Plástico \\
\hline 01 & Chassi composto por três placas de Acrílico \\
\hline 01 & Resistor de 330 $\Omega$ \\
\hline 01 & Módulo Bluetooth HC-06 \\
\hline 01 & Sensor Ultrassônico \\
\hline 01 & Cabo USB para alimentar Arduino \\
\hline 01 & Conjunto de 8 pilhas AA para alimentar motores \\
\hline 01 & Bateria Estacionária 12V \\
\hline 01 & Kinect Xbox360 \\
\hline 01 & Notebook (Core i3 2377M e 8GB de RAM) \\
\hline 01 &
\end{tabular}

Na Figura 3 é apresentado o RecArd. O mesmo é constituído de três plataformas de acrílico: A maior e primeira (chassis 1), de cima para baixo, contém o notebook e Kinect; a segunda plataforma de acrílico (chassis 2, na cor preta), fica as baterias e módulo bluetooth; e na terceira e última (chassis 3), fica o circuito principal, composto pelo Arduino e Shield, além das rodas que são parafusadas neste último chassi.
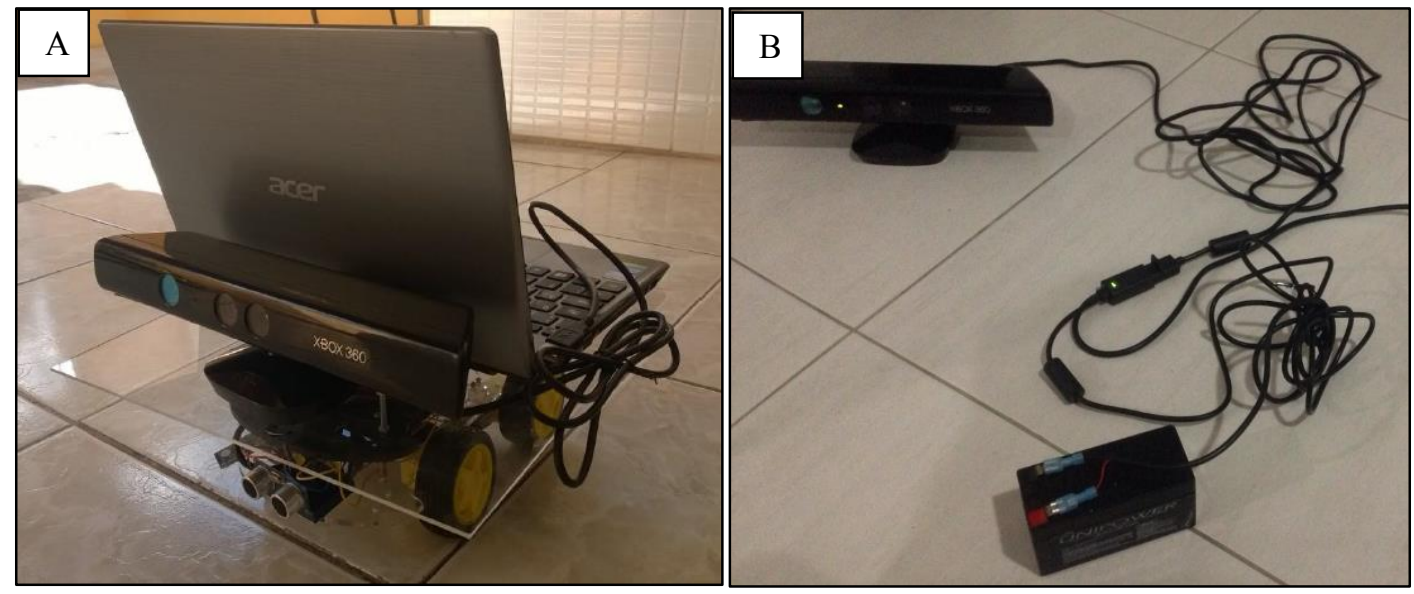

Figura 3. (A) Robô RecArd e (B) Kinect com Bateria 12V.

É válido destacar que o robô pode ser conectado via bluetooth com o módulo HC06 e através do aplicativo BT Terminal do Windows Phone pode ser controlado por 
comandos. O comando "w" desloca o robô para frente, "s" desloca para trás, "a" para esquerda, "d" para direita e " $\mathrm{x}$ " interrompe os motores. Ressalta-se também que a conexão do robô pode ser feita com outros modelos de smartphone, inclusive com outros sistemas, como o Android e iOS. Este modo de operação foi utilizado durante as apresentações de minicursos e em sala de aula para interação do estudante com o projeto.

Após a construção deste protótipo, foi iniciada a fase da análise do projeto. A análise foi realizada em duas etapas: A primeira consistiu na utilização do prótotipo em ambiente fechado para avaliar seu desemprenho quanto a reconstrução de objetos. Já a segunda etapa, utilizou-se a Escala de Concordância Likert de um a cinco, em que a ordem crescente desses numeros indicam de péssimo à excelente, respectivamente. Esta ultima foi preenchida por estudantes e professores após as apresentações do protótipo.

\section{Resultados e Discussão}

A presente seção aborda a inserção da Reconstrução 3D e o uso da robótica na educação bem como sua importância para, posteriormente, relatar e apresentar os resultados coletados com o projeto a partir das atuais pesquisas e debates em sala de aula.

\subsection{Reconstrução 3D voltado à Educação}

Durante a realização do projeto, percebeu-se que a Reconstrução 3D poderia ser aplicada para o estudo de conteúdos ministrados em sala de aula no curso de Engenharia Elétrica. Neste caso, foi realizado a reconstrução tridimensional de isoladores elétricos, ora em desuso, pertencentes a Companhia Hidrelétrica do São Francisco (CHESF).

Para a realização da reconstrução, os isoladores foram colocados em uma base e foi realizado a reconstrução normalmente usando o robô com o Kinect. Após o processo, foi feito a edição das imagens 3D para remover a base colocada, utilizando o Meshlab. A Figura 4 mostra os isoladores reconstruídos tridimensionalmente.
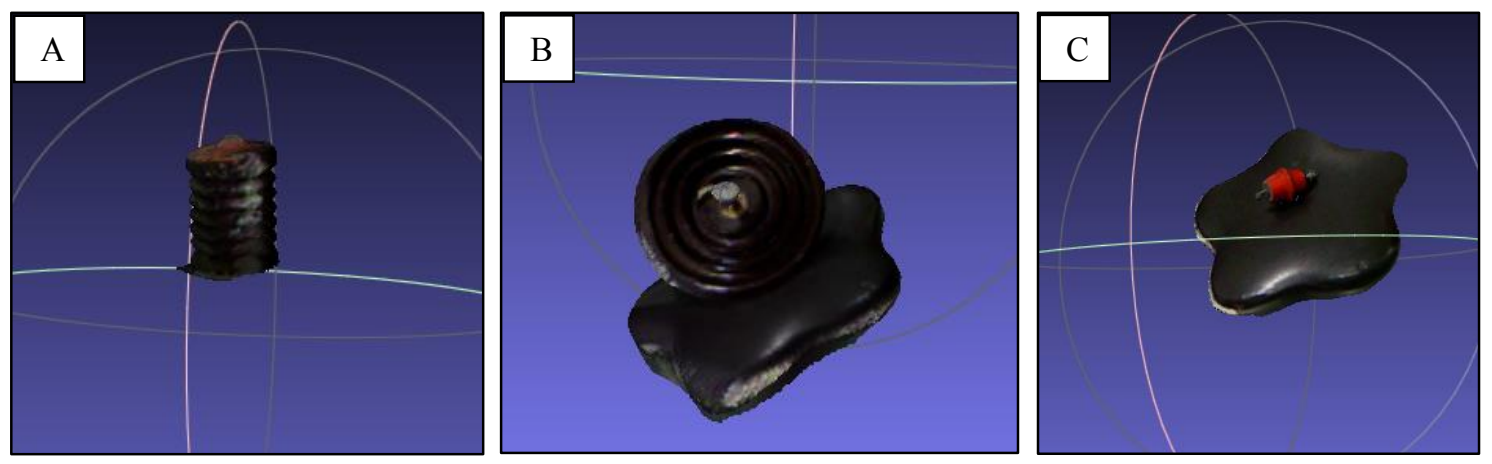

Figura 4. Reconstrução de isolador tipo (A) Pedal, (B) Singelo e (C) Bujão.

Com o uso das Reconstruções pode-se mostrar, de uma melhor forma, alguns equipamentos usados na área de Engenharia Elétrica. Logo, a reconstruçao 3D permite a visualização de equipamentos e instrumentos, de forma geral, de uma maneira interativa e construtiva. Isso proporciona, consequentemente, um ambiente mais divertido e mais compreensivel aos estudantes, visto que os mesmo tem o contato com isoladores de forma virtual. 


\subsection{Utilização do Protótipo em um ambiente fechado}

Para realização de outro teste, neste caso envolvendo a localização de objetos, o protótipo foi colocado dentro de uma sala com dimensão de $25 \mathrm{~m}^{2}$ e com obstáculos dispersos para dificultar a movimentação do robô. Em determinado lugar da sala foi colocado um jarro, onde o mesmo possui diversos detalhes em sua forma - o que dificulta a atuação do Kinect na reconstrução 3D. Antes do teste, também foi adicionado uma foto do objeto na biblioteca do Find-Object, que pode ser definida como uma interface QT designada a implementações OpenCV para detectar características de forma rápida [Labbé 2015].

O Robô conseguiu desviar dos obstáculos com a ajuda dos sensores ultrassónicos até chegar ao objeto e localiza-lo com o Find-Object. Após isso, fez-se uma reconstrução 3D parcial do objeto como apresentada na Figura 5. Foi realizado acesso remoto para acompanhar e obter as telas, a Figura 5 (b) já é a reconstrução editada no MeshLab.
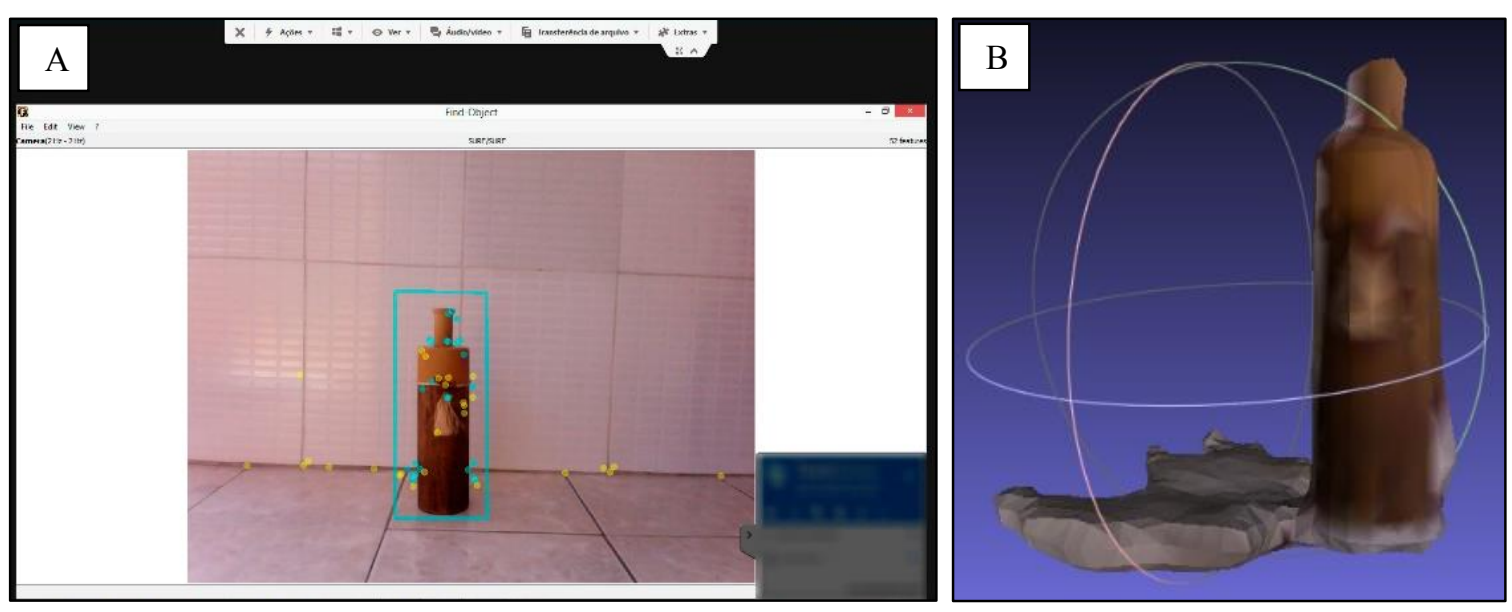

Figura 5. Objeto (a) Real sendo localizado e (b) Reconstruído tridimensionalmente.

Ressalta-se que o Kinect possui limitações e dificuldades para reconstruir detalhes. No caso do vaso, o mesmo, que apesar de ter um contorno que facilita a reconstrução 3D, ele tem linhas e desenhos em sua estrutura que dificulta bastante a reconstrução. Além disso, deve-se levar em consideração seu pequeno tamanho $(28 \mathrm{~cm}$ de altura). Entretanto, o principal fator limitador da reconstrução foi a configuração do notebook, visto que, sua placa de vídeo dificultou ainda mais o processo.

\subsection{Análise dos Resultados obtidos com a aplicação educacional}

O protótipo do robô foi apresentado na forma de minicurso, para avaliação do desempenho do mesmo quanto ao seu uso educacional. Para tanto, foi ativado o controle por bluetooth e removido o Kinect, deixando assim ele mais interativo para os estudantes controlar, já que o objetivo principal era ensinar programação, eletrônica e mecânica com o protótipo, e não a captura de objetos pelos alunos, inicialmente.

Em primeira análise, percebeu-se o potencial atrativo que experimentos como esse pode vim a trazer para alunos de diversas áreas, não contando somente, neste caso, áreas especificas como o que pretende-se avaliar no presente trabalho, sendo elas engenharia e computação. 
Com o desenvolvimento do Robô e com o material para o minicurso, foi perceptível que o projeto poderia discutir diversas questões como: "Definir sensores e atuadores e qual a diferença entre eles?", "O que é e qual importancia de usar a Ponte H?", "Por que o LED necessita de um resistor?", "Como funciona o sensor ultrassônico?", “Como é possível o RecArd obedecer aos comandos?”, "Como determinar a voltagem para alimentar o robô?", entre outros.

Outro fato marcante na análise educacional do projeto foi o nível de questionamentos e direcionamentos a áreas, principalmente, de engenharia e computação, levantados em questão, principalmente pelos alunos de Engenharia Elétrica e Curso Técnico em Informática, pois, professores e curiosos também participaram do minicurso e tiveram suas contribuições quanto ao projeto.

Em um dos depoimentos um aluno afirmou "O projeto diminui a distância entre o aluno e a tecnologia aplicada fazendo com que ele se sinta ambientado com tal, além de diminuir a abstração de diversos assuntos". Outro aluno declarou "A robótica educacional ajuda a levar o aluno a questionar, pensar e procurar soluções, a sair da rotina de sempre teoria e ir para a prática. Desperta curiosidade e um grande interesse". Ambos comentários confirmaram, mesmo que não provasse de forma concreta, o idealizado e esperado pelo projeto.

Ademais, um comentário relatou também a importância da robótica para o estudante, pois a mesma faz com que o discente obtenha conhecimentos diversificados: "Para se entender o que está "por trás da robótica" é necessário aprender e entender conceitos voltados para diversas áreas de exatas, como programação, engenharia elétrica e mecânica, entre outras. Desta forma, um estudante que busque essas áreas, terá conhecimentos vastos e diversificados".

Em suma, a pesquisa indicou que os discentes (público alvo da pesquisa), em sua grande maioria, consideraram a robótica (o robô) como um estímulo de estudo e como um mecanismo positivo para auxiliar na didática dos professores. Além disso, foi mencionado que o projeto poderia levar os discentes a se distraírem, tirando o foco do ensino.

Entretanto, com um levantamento mais apurado e com o objetivo de averiguar o nível de concordância e aceitação dos estudantes sobre a implantação do projeto, obtevese resultados positivos como mostrado na Figura 6, ilustrada de acordo com a escala de concordância, chamada de Escala Likert, com 5 notas no formato ordinal: (5) Excelente, (4) Bom, (3) Regular, (2) Ruim e (1) Péssimo. Resultados obtidos através de um questionário eletrônico enviado aos alunos, e respondido por 43 destes. 


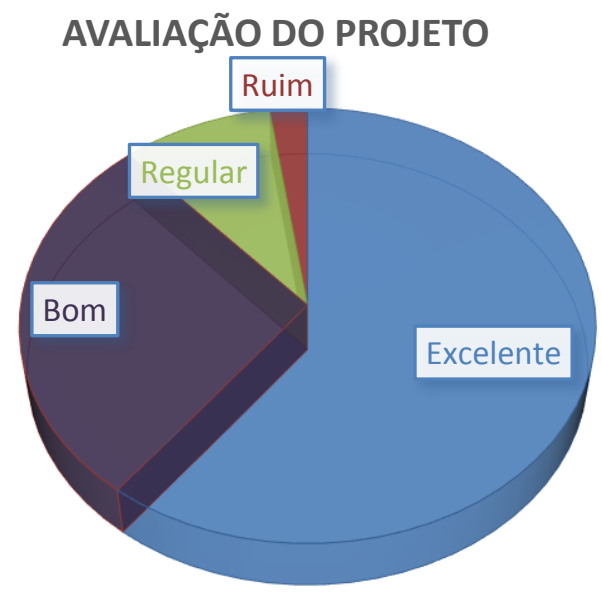

Figura 6. Resultados na escala de concordância para o projeto

O gráfico foi montado com os seguintes resultados: 60.46\% (26 pessoas) dos participantes atribuíram a pontuação máxima ao projeto, $27.91 \%$ (12 pessoas) nota quatro, $9.30 \%$ (4 pessoas) nota três e $2.33 \%$ (1 pessoa) atribuíram nota dois. Além disso, foi recolhido e considerado diversas opiniões a respeito do projeto, a ser levada em consideração na melhoria do protótipo inicial.

\section{Considerações finais}

Com o projeto pode-se acrescentar outras habilidades e competências que permite que os alunos compreendam melhor as disciplinas nas áreas de eletrônica e computação principalmente, e, assim, obtenham um melhor desempenho nas mesmas. Foi possível sentir um estímulo maior na busca do conhecimento quando conhece a aplicação prática de determinados conteúdo, isto é, o estudo une a teoria e a prática e assim faz com que dúvidas sejam solucionadas, além mostrar a união do hardware e software e do uso dos componentes eletrônicos, o que torna o protótipo algo multidisciplinar.

No aspecto da reconstrução tridimensional percebeu-se que existe a necessidade de utilizar um notebook com uma melhor placa de vídeo, como também é necessário um chassi com motores mais potentes e com uma roda livre e duas com motores, ao invés das quatro rodas. Desta forma, tem-se um protótipo com mais agilidade na realização de curvas, o que é importante para contornar objetos durante a reconstrução tridimensional. É ainda necessário relatar que o uso de realidade virtual e aumentada em educação é um desafio a ser superado, devido principalmente as dificuldades em usar recursos computacionais por parte dos docentes [Martins 2012].

Foi perceptível também a importância dos modelos 3D, quando utilizados de forma educacional, a reconstruçao $3 \mathrm{D}$ permite a visualização de equipamentos e instrumentos, de forma geral, o que permite o aluno ter contato mais próximo de equipamentos que só poderia ser vistos por fotos, assim tem-se uma maneira mais lúdica e interativa de mostrar diversos materiais.

Como trabalhos futuros, pretende-se aprimorar o robô e deixá-lo mais interativo, incluindo a ele outros sensores e equipamentos, destinando o foco a educação, 
planejando-se ministrar palestras e minicursos para divulgar o trabalho e para estender o conhecimento e atividades multidisciplinares a comunidade. Em conjunto, pretende-se buscar formas de melhorar o aspecto da reconstrução tridimensional, buscando corrigir os problemas e aspectos vistos com a pesquisa no protótipo.

\section{Referências}

Barros R. P.; Torres V. P.; Burlamaqui A. M. F. (2014) "CardBot: Tecnologias assistivas para imersão de deficientes visuais na robótica educacional”, In: Workshop de Robótica Educacional. Anais do $5^{\circ}$ Workshop de Robótica Educacional - WRE 2014, São Carlos - SP, 2014.

Cardoso, G. S. (2013) "Microsoft Kinect: Crie aplicações interativas”, Edição 1ª Local de publicação: Editora Casa do Código,p. 167, 2013.

Cassol, P. B. (2000) "Globalização e educação: elementos para repensar a atuação do professor face as mudanças tecnológicas no atual context”, 2000.

Cavalcante, M.M; Silva, J. L. S.; Cabral, E. V.; Dantas, J. R. A. (2014) "Plataforma Arduino pra fins didáticos: Estudo de caso com recolhimento de dados a partir do PLXDAQ”, In: Congresso da Sociedade Brasileira de Computação, 2014, Brasília. Anais. Brasília: Sociedade Brasileira de Computação, p.1655-1664.

Labbé, M. (2015) "Find-Object Project", https://code.google.com/p/find-object/, Fevereiro.

Lévy, P.; Da Costa, (1993) C. I. tecnologias da inteligência, As. Editora 34, 1993.

Martins, V. F.; Guimarães, M. P. (2012) "Desafios para o usode Realidade Virtual e Aumentada de maneira efetiva noensino", In: DEsafIE! - I Workshop de Desafios da Computação Aplicada à Educação - XXXII Congresso da Sociedade Brasileira de Computação, 2012, Curitiba. Anais do DEsafIE!.

Parpet, S. A. (2008) "Máquina das crianças: repensando a escola na era da informática", Tradução de Sandra Costa. Porto Alegre: Artes Médicas, 2008. p.210.

Santos, F.L.; Nascimento, F. M. \& Bezerra, R. A. (2010) "Robótica Educacional como Abordagem de Baixo Custo para o Ensino de Computação em Cursos Técnicos e Tecnológicos", In: Congresso da Sociedade Brasileira de Computação, 2010, Belo Horizonte. Anais. Belo Horizonte: Sociedade Brasileira de Computação, p.1304-1313.

Silva, J. L. S.; Cavalcante, M. M.; Vaz, F. A.; Dantas, J. R.; Cabral, E. V. (2014a) RecArd: Robô baseado na plataforma Arduino como facilitador no processo de ensinoaprendizagem multidisciplinar. Revista Novas Tecnologias na Educação (RENOTE). vol. 12, n. 2, 2014a.

Silva, J. L. S.; Santos, R. S.; Vaz, F. A. (2014b) "O Kinect Fusion como técnica de reconstrução tridimensional de objetos em tempo real visando a construção de ambientes virtuais multissensoriais", In: IX Congresso Norte Nordeste de Pesquisa e Inovação (Connepi), 2014, São Luís - MA. Anais do IX Congresso Norte Nordeste de Pesquisa e Inovação (Connepi), 2014b. 